\title{
Connecting Peripheral to Central Neuropathy: Examination of Nerve Conduction Combined with Olfactory Tests in Patients with Type 2 Diabetes
}

\author{
Wenyu $\mathrm{Ni}^{1, *}$ \\ Zhou Zhang ${ }^{1, *}$ \\ Bing Zhang ${ }^{2}$ \\ Wen Zhang ${ }^{2}$ \\ Haiyan Cheng' \\ Yingwen Miao' \\ Wei Chen' \\ Jiani Liu $^{2}$ \\ Dalong Zhu' \\ Yan $\mathrm{Bi}^{1}$
}

'Department of Endocrinology, Nanjing Drum Tower Hospital, The Affiliated Hospital of Nanjing University Medical School, Nanjing, People's Republic of China; ${ }^{2}$ Department of Radiology, Nanjing Drum Tower Hospital, The Affiliated Hospital of Nanjing University Medical School, Nanjing, People's Republic of China

*These authors contributed equally to this work

\begin{abstract}
Aim: Few studies have investigated the associations between diabetic peripheral neuropathy (DPN) and cognitive decline. Olfactory impairment is related to neurodegenerative diseases and type 2 diabetes mellitus (T2DM); however, the cognitive alterations of patients with DPN and the role of olfactory function in DPN are not known. We explored alterations in cognition with DPN and the associations of neuropathy parameters with cognition and olfaction.
\end{abstract}

Methods: Healthy controls (HCs) and patients with T2DM underwent nerve-conduction tests, detailed cognitive assessment, olfactory-behavior tests, and odor-induced functional magnetic resonance imaging (fMRI). T2DM patients were divided into two groups (nonDPN [NDPN] and DPN). Olfactory brain regions showing different activation between the two groups were selected for functional connectivity (FC) analyses. A structural equation model (SEM) was also generated to demonstrate the association among cognition, olfactory, and neuropathy parameters.

Results: One hundred individuals (36 HCs, $36 \mathrm{NDPN}$, and $28 \mathrm{DPN}$ ) were matched for age, sex, and educational level. Compared with the NDPN group, the DPN group had significantly lower scores for memory and processing speed, as well as lower olfactory identification and memory scores, decreased activation of the left frontal lobe, and reduced seed-based functional connectivity in the right insula. The nerve conduction velocity in patients with T2DM was associated with cognitive functions. The association between nerve conduction and executive function was mediated by olfactory behavior.

Conclusion: Patients with DPN had worse cognition than the NDPN patients in the domains of memory and processing speed. Cognitive dysfunction could be predicted by olfactorybehavior tests and electrophysiological examination.

Keywords: type 2 diabetes mellitus, peripheral neuropathy, cognitive dysfunction, olfactory behavior, odor-induced brain activation

\section{Introduction}

The increase in the size of the global aging population and the prevalence of type 2 diabetes mellitus (T2DM) have led to grave individual, societal, and financial burdens associated with diabetes-related cognitive dysfunction. ${ }^{1,2}$ Studies have indicated that individuals suffering from diabetes have a 1.5-to-2.5-times increased risk of cognitive decline, ${ }^{3}$ and the dementia progression cannot be reversed once clinical symptoms
Department of Endocrinology, Drum Tower Hospital, Affiliated to Nanjing University Medical School, No. 32I,

Zhongshan Road, Nanjing, 210008,

People's Republic of China

Tel +86 25-833046|6-6 I43I

Fax +86 25-833046|6-6I43।

Email biyan@nju.edu.cn 
occur. $^{4}$ Thus, early diagnosis is beneficial for delaying disease progression and improving prognosis.

Diabetic peripheral neuropathy (DPN) occurs in up to $50 \%$ of patients with type 2 diabetes, ${ }^{5}$ and can lead to disability and amputation; therefore, it is associated with poor quality of life and high mortality. ${ }^{6-8}$ However, the relationship between DPN and cognitive impairment is not clear. One study observed no significant differences in cognitive function between patients with and without DPN, ${ }^{9}$ whereas a follow-up study found that DPN carried a 1.61-times higher risk of the development of mild cognitive impairment (MCI). ${ }^{10}$ Therefore, assessing the clinical features of cognitive decline in patients with DPN merits investigation.

Olfactory tests have potential utility for screening for $\mathrm{MCI},{ }^{11}$ as olfactory impairment is related to neurodegenerative diseases and T2DM. ${ }^{11,12}$ Typically, olfactory dysfunction occurs at 4-8 years before the diagnosis of Parkinson's disease, ${ }^{13}$ and low scores in olfactory tests suggest an increased probability of a diagnosis of Alzheimer's disease over the subsequent $2-5$ years. ${ }^{14}$ We previously revealed that the olfactory threshold scores were lower in patients with T2DM than in people not suffering from T2DM, and that alterations in olfactory behavior occurred before clinically measurable cognitive decrements in T2DM. ${ }^{15}$ The application of magnetic resonance imaging (MRI) and functional MRI (fMRI) have confirmed the reductions in odor-induced brain activation and the weakened functional connectivity (FC) in the olfactory network in patients with T2DM. ${ }^{15,16}$ However, alterations in the olfactory network of patients with DPN have not been investigated.

In the present study, we evaluated the cognitive function, olfactory behavior, and activation of olfactory-related brain functions in healthy controls (HCs), T2DM patients without DPN, and T2DM patients with DPN. This allowed us to explore the clinical features of cognitive decline and the role of olfactory-circuit alterations on cognitive dysfunction in T2DM patients with DPN.

\section{Methods}

\section{Subjects}

This study was carried out from August 2017 to August 2019 at Drum Tower Hospital, The Affiliated Hospital of Nanjing University Medical School. The HCs and patients with T2DM were recruited. T2DM was diagnosed according to the criteria set by the World Health Organization/International Diabetes Federation. The data of 100 individuals (64 T2DM patients and $36 \mathrm{HCs}$ ) matched for age, sex, and educational level were analyzed. The inclusion criteria were patients: (i) aged from 40 to 75 years; (ii) right-handed; and (iii) with over 6 years of education. The exclusion criteria were patients: (i) with neurological and psychiatric disorders, anxiety or depression; (ii) with a history of frequent hypoglycemic episodes, cerebrovascular disease or cardiovascular disease, corticosteroid treatment, abnormal thyroid function, alcohol/substance abuse, infections or cancer; (iii) with nondiabetic peripheral neuropathies: cervical spine and lumbar disease, connective tissue disease, inflammatory neurologic diseases, or exposure to peripheral neurotoxicity of chemicals; (iv) with nasal diseases affecting olfactory function (eg, allergic rhinitis, acute/chronic sinusitis, deviated nasal septum, or nasal polyposis); (v) without the ability to undergo olfactory tests or MRI; (vi) with excessive head movement during fMRI $\left(>2.5^{\circ}\right.$ rotation or $>2.5 \mathrm{~mm}$ shift and image artifacts).

\section{Neurological Assessment}

A system from Nihon Kohden (Tokyo, Japan) was used for electromyographic measurements of the nerve conduction velocity (NCV) by a very experienced technician. The skin temperature of the patients was stabilized to approximately $31^{\circ} \mathrm{C}$ at a room temperature of $24^{\circ} \mathrm{C}$. The $\mathrm{NCV}$ was assessed on both sides: median nerve (motor and sensory), ulnar nerve (motor and sensory), common peroneal nerve (motor), tibial nerve (motor), and sural nerve (sensory). Nerve conduction was considered "abnormal" if $\geq 3$ of the tested NCVs were reduced below the reference value specific to the Chinese population. ${ }^{17}$ Abnormalities of nerve conduction and the symptoms or signs of neuropathy were used to confirm DPN. ${ }^{6}$ The DPN symptoms included neuropathic sensory symptoms, mainly in the legs, feet, or toes, as well as numbness, prickling, burning, or aching pain. DPN signs included symmetrical reduction in distal sensation or decreased/absent ankle reflexes.

\section{General Cognition and Tests of Cognitive Domains}

The Mini-Mental State Examination (MMSE) and Montreal Cognitive Assessment (MoCA; Beijing Version) were used to evaluate general cognition. ${ }^{18}$ A MoCA score of 19-25 was regarded as evidence of mild cognitive impairment. Multiple cognitive subdomains were also assessed. The 16word Philadelphia Verbal Learning and Wechsler Memory 
Scale tests Trail Making Test (Parts A and B), Animal Naming Test, Boston Naming Test, Stroop Color-Word Test (Parts I, II and III), and Digit Span Test (forward and backward) were conducted to evaluate episodic memory, information-processing speed, word fluency, executive function, and working memory. All tests needed approximately 60 minutes to complete in a fixed order.

\section{Tests of Olfactory Behavior}

Olfactory tests were carried out using the Olfactory Function Assessment by Computerized Testing $\left(\right.$ OLFACT $\left.^{\mathrm{TM}}\right)$ with a system from Osmic Enterprises (Cincinnati, OH, USA), as described previously. ${ }^{16}$ The olfactory tests were computerized and standardized. The olfactory threshold test was conducted using a series of binary dilutions of an n-butanol solution in light mineral oil. The scores indicated the sensitivity of participants to detect odors, and the values ranged from 1 to 13.5. The olfactory identification test and memory test had two tasks: task A had 10 odors, while task B had the same 10 odors as task A, as well as 10 new odors. Participants were exposed to the odors and were required to identify each one from four pictures in the two tasks. In task B, they also had to indicate whether each odor was new or old. Participants had a 10-min break between tasks A and B.

\section{Clinical Assessment}

Clinical data were collected using a standardized questionnaire that included information on demographics, T2DM duration, alcohol consumption and smoking, history of stroke, and family history of T2DM, as well as measurements of height, weight, waist circumference, hip circumference, and resting blood pressure. Patients with T2DM underwent a $100 \mathrm{~g}$ standard meal test. Individuals without previously diagnosed T2DM were subjected to a $75 \mathrm{~g}$ oral glucose tolerance test after an overnight fast of at least 8 h. Plasma concentrations of glucose, insulin, and C-peptide were measured upon fasting and $2 \mathrm{~h}$ after glucose ingestion. Concentrations of glycosylated hemoglobin $\left(\mathrm{Hb}_{\mathrm{Alc}}\right)$, total cholesterol (TC), triglyceride (TG), highdensity lipoprotein-cholesterol (HDL-C), and low-density lipoprotein cholesterol (LDL-C) were also measured.

\section{Odor-Induced fMRI Paradigm}

Our previous study utilized the odor-induced fMRI paradigm, ${ }^{15}$ which consists of 12 trials. Every trial comprised 30 seconds of odorless fresh air and 6 seconds of odor stimulation with lavender oil (Givaudan Flavors, East Hanover, NJ, USA). Four gradually increasing concentrations of oil $(0.032 \%, 0.10 \%, 0.32 \%$, and $1.0 \%)$ were diluted in 1,2-propanediol (Sigma-Aldrich, Saint Louis, MO, USA) to offset the effect of brain habituation. Each concentration was used three times, alternating between fresh air and lavender scent. Throughout the fMRI, the technician asked participants to keep breathing and press the button once they smelled the lavender scent.

\section{MRI Acquisition}

Image data were acquired on a 3.0T MRI scanner (Achieva 3.0T TX, Philips Medical Systems, The Netherlands) with an 8-channel head coil. T1-weighted images were acquired with $\mathrm{TR} / \mathrm{TE}=9.7 \mathrm{~ms} / 4.6 \mathrm{~ms}, \mathrm{FA}=8^{\circ}, \mathrm{FOV}=256 \mathrm{~mm} \times 256 \mathrm{~mm} \times$ $192 \mathrm{~mm}$, voxel size $=1 \mathrm{~mm} \times 1 \mathrm{~mm} \times 1 \mathrm{~mm}$. Functional MRI was acquired with a gradient-echo planar imaging sequence scan, TR $/ \mathrm{TE}=2300 \mathrm{~ms} / 30 \mathrm{~ms}, \mathrm{FA}=90^{\circ}, \mathrm{FOV}=192 \mathrm{~mm} \times$ $192 \mathrm{~mm} \times 140 \mathrm{~mm}$, slice thickness $=4 \mathrm{~mm}$, voxel size $=3 \mathrm{~mm} \times 3 \mathrm{~mm} \times 4 \mathrm{~mm}$, with 230 repetitions for resting-state fMRI and 222 repetitions for odor-induced fMRI.

\section{Image Preprocessing}

Statistical Parametric Mapping (SPM12, Wellcome Trust Centre for Human Neuroimaging, London, UK) was used to preprocess the fMRI data. SPM12 was undertaken in six distinct steps. In step 1, the first six images of the "task" scan and the first 10 images of the "resting state" scan were discarded to remove fluctuations in the initial transit signal. In step 2, spatial realignment was done to remove head movements: shift $<3 \mathrm{~mm}$ and rotation $<3^{\circ}$. Step 3 consisted of co-registration with the T1-weighted high-resolution anatomical image. Step 4 was spatial normalization to the brain template of the Montreal Neurological Institute. Step 5 was spatial smoothing with an 8-mm Gaussian smoothing kernel. Step 6 was linear detrending and temporal band-pass filtering $(0.01-0.08 \mathrm{~Hz})$ to eliminate high-frequency noise and low-frequency drift.

\section{Brain Activation Analysis}

Brain activation during the olfactory-stimulation tasks was estimated using a general linear model. Each participant had three conditions in the fMRI task: "fresh air," "scent," and "rest." Contrasts were made between every two conditions. We chose several olfactory-related regions as our regions of interest (ROI); these covered the hippocampus, bilateral parahippocampus, piriform cortex, amygdala, insula, and orbitofrontal cortex in the Automated Anatomical Labeling templates and Brodmann areas 28 and 34 (entorhinal 
cortices). The total cluster size was 5029 voxels. Differences in activations between groups were estimated as beta values.

\section{FC Analyses}

FC was computed using a seed-based correlation analytical method. Seed regions were selected as brain regions that showed significantly different activation during olfactory stimulation in patients with DPN when compared with patients not suffering from DPN. We then extracted the time series of the signal in the seed regions and used these time series to generate voxel-wise FC maps.

\section{Statistical Analyses}

Data for continuous variables are presented as the mean \pm standard deviation and dichotomous variables as percentages. One-way analysis of variance (ANOVA), followed by Dunnett's test, was conducted for continuous variables. Pearson chi-square was performed for dichotomous variables. Independent-samples t-tests were used to compare the duration in both diabetes groups. Partial correlation analyses, controlled for age, sex, and educational level, were conducted to analyze the association of the NCV with olfactory behavior and cognitive function. Differences were considered statistically significant when $\mathrm{P}$ was $<0.05$. These analyses were undertaken with SPSS v20.0 (IBM, Armonk, NY, USA).

DPABI (v4.1_190725) was used to analyze the fMRI data. Differences in brain function were determined using voxel-based independent-sample $t$-tests for odor-induced brain activation and seed-based FC of the resting state between two groups. Correction of the significance threshold was based on Gaussian random field (GRF) theory, with a voxel level of $\mathrm{P}<0.001$ and a cluster level of $\mathrm{P}<0.05$.

Structural equation modeling (SEM) using SPSS Amos version 24 was conducted to determine the interrelationships of nerve conduction, the olfactory system, and cognitive function. The mean- and variance-adjusted maximum likelihood test statistic (MLMV) was performed for the SEM, given its robustness for data with non-normal distribution. Root mean square error of approximation (RMSEA; good if $\leq 0.08$ ); normal fit index (NFI $>0.90$ ); comparative fix index (CFI $>0.90)$; and Tucker-Lewis index (TLI $>0.90)$ were used to assess the global structural fitness of the model.

\section{Results}

\section{Clinical Characteristics, Cognition Status, and Olfactory Behavior of DPN}

Overall, 100 individuals matched for age, sex, and educational level were analyzed: 36 T2DM patients without DPN (NDPN group), 28 T2DM patients with DPN (DPN group), and $36 \mathrm{HCs}$. The T2DM patients had higher $\mathrm{Hb}_{\mathrm{A} 1 \mathrm{c}}$ levels, fasting and two-hour plasma glucose levels, waist circumference, and waist-to-hip ratios, and lower postprandial C-peptide levels when compared with the HCs. The DPN group had higher $\mathrm{Hb}_{\mathrm{Alc}}$ levels than the NDPN group. No significant differences were noted in the TG or TC levels. The motor conduction velocity (MCV) of the median nerve, ulnar nerve, common peroneal nerve, and tibial nerve and the sensory conduction velocity (SCV) of the median nerve, ulnar nerve, and sural nerve were slower in the DPN patients than in the NDPN cases and HCs (Table 1).

The MMSE, MoCA score, memory, processing speed, working memory, and executive function were all significantly lower in the DPN patients than in the HCs. Compared with the NDPN group, the DPN group had a significantly lower score for memory $(155.2 \pm 15.2$ vs $142.0 \pm 20.0, p=0.042)$ and a longer time required for the processing speed test $(102.3 \pm 37.9$ vs $134.2 \pm 69.2$, $p=0.004)$ (Table 1).

Olfactory behavior, including odor identification and memory, was decreased in the DPN patients compared with the NDPN patients. The DPN group had a significantly lower score than the HCs for the olfactory threshold and odor identification and memory (Table 1).

\section{Associations of Cognitive Function and Olfactory Behavior with Neuropathy Parameters}

Correlation analyses among patients with T2DM showed that memory had a positive association with the MCV and SCV of the ulnar nerve. Time spent in executive function and the processing speed test had a negative association with the MCV and SCV of the ulnar nerve. Higher olfactory identification and memory scores were correlated with faster SCVs of the median nerve (Table 2). 
Table I Demographics, Clinical and Metabolic Characteristics, Cognitive Assessment Scores, and Olfactory Test Scores of T2DM Patients by DPN Status

\begin{tabular}{|c|c|c|c|c|c|}
\hline & HCs & NDPN & DPN & \multirow{2}{*}{$\begin{array}{c}P \text { value of } \\
\text { All }\end{array}$} & \multirow{2}{*}{$\begin{array}{l}P \text { value of } \\
\text { Diabetes }\end{array}$} \\
\hline & $n=36$ & $n=36$ & $\mathbf{n}=\mathbf{2 8}$ & & \\
\hline \multicolumn{6}{|l|}{ Demographic factors } \\
\hline Age (years) & $52.0 \pm 8.1$ & $53.1 \pm 8.1$ & $53.9 \pm 8.3$ & 0.632 & 0.684 \\
\hline Sex $($ male, $n, \%){ }^{\#}$ & 17,47 & 19,53 & 16,57 & 0.728 & 0.803 \\
\hline Education (years) & $12.3 \pm 3.2$ & $11.6 \pm 2.5$ & $12.0 \pm 3.0$ & 0.580 & 0.592 \\
\hline Alcohol consumption (\%) ${ }^{\#}$ & 9,25 & 10,28 & 8,29 & 0.942 & 1.000 \\
\hline Smoking habits (\%) & 12,33 & 14,38 & 11,39 & 0.752 & 1.000 \\
\hline \multicolumn{6}{|l|}{ Diabetes-related characteristics } \\
\hline Duration of diabetes (years) & - & $9.8 \pm 6.3$ & $11.5 \pm 6.7$ & - & 0.287 \\
\hline $\mathrm{HbA}_{\mathrm{Ic}}(\%)$ & $5.6 \pm 0.1$ & $8.3 \pm 1.7^{\mathrm{a}}$ & $9.2 \pm 1.9^{b c}$ & 0.000 & 0.042 \\
\hline Fasting glucose (mmol/L) & $5.0 \pm 0.4$ & $8.3 \pm 2.6^{\mathrm{a}}$ & $7.8 \pm 2.5^{\mathrm{b}}$ & 0.027 & 0.424 \\
\hline $2 \mathrm{~h}$ postprandial glucose $(\mathrm{mmol} / \mathrm{L})$ & $6.6 \pm 1.5$ & $14.5 \pm 4.9^{\mathrm{a}}$ & $13.9 \pm 5.2^{b}$ & 0.005 & 0.647 \\
\hline Fasting insulin (mlU/mL) & $7.0 \pm 2.1$ & $11.8 \pm 8.6$ & $11.2 \pm 18.8$ & 0.773 & 0.877 \\
\hline $2 \mathrm{~h}$ postprandial insulin (mIU/mL) & $62.6 \pm 35.4$ & $37.6 \pm 35.8$ & $37.1 \pm 78.8$ & 0.705 & 0.973 \\
\hline Fasting C-peptide (pmol/L) & $630.6 \pm 167.1$ & $754.1 \pm 424.2$ & $576.8 \pm 376.5$ & 0.209 & $0.08 \mathrm{I}$ \\
\hline 2h postprandial C-peptide (pmol/L) & $2933.6 \pm 1651.3$ & $1748.2 \pm 901.3$ & $1287.1 \pm 1388.4^{b}$ & 0.044 & 0.123 \\
\hline \multicolumn{6}{|l|}{ Clinical variables } \\
\hline $\mathrm{BMI}(\mathrm{kg} / \mathrm{m} 2)$ & $23.9 \pm 2.7$ & $26.2 \pm 3.7^{\mathrm{a}}$ & $25.1 \pm 3.6$ & 0.025 & 0.208 \\
\hline Waist $(\mathrm{cm})$ & $84.1 \pm 6.8$ & $92.8 \pm 9.4^{\mathrm{a}}$ & $90.8 \pm 11.7^{b}$ & 0.001 & 0.406 \\
\hline WHR & $0.8 \pm 0.1$ & $0.9 \pm 0.1^{\mathrm{a}}$ & $0.9 \pm 0.1^{b}$ & 0.000 & 0.260 \\
\hline Systolic Blood Pressure $(\mathrm{mmHg})$ & $123.3 \pm 16.6$ & $134.2 \pm 15.5^{\mathrm{a}}$ & $129.2 \pm 15.8$ & 0.020 & 0.220 \\
\hline Diastolic Blood Pressure $(\mathrm{mmHg})$ & $79.3 \pm 14.5$ & $82.1 \pm 10.6$ & $80.3 \pm 12.9$ & 0.645 & 0.564 \\
\hline Triglycerides (mmol/L) & $1.5 \pm 0.5$ & $1.9 \pm 0.9$ & $1.9 \pm 1.2$ & 0.308 & 0.963 \\
\hline Total cholesterol $(\mathrm{mmol} / \mathrm{L})$ & $4.8 \pm 0.8$ & $4.7 \pm 1.3$ & $4.8 \pm 0.9$ & 0.710 & $0.50 \mathrm{I}$ \\
\hline HDL cholesterol $(\mathrm{mmol} / \mathrm{L})$ & $1.2 \pm 0.4$ & $1.1 \pm 0.3^{\mathrm{a}}$ & $1.2 \pm 0.4$ & 0.075 & 0.073 \\
\hline LDL cholesterol (mmol/L) & $2.7 \pm 0.7$ & $2.9 \pm 1.2$ & $2.9 \pm 0.8$ & 0.964 & 0.951 \\
\hline \multicolumn{6}{|l|}{ Neuropathy parameters } \\
\hline \multicolumn{6}{|l|}{$\mathrm{MCV}$} \\
\hline Median nerve & $57.6 \pm 2.6$ & $55.6 \pm 3.5$ & $50.6 \pm 6.3^{b c}$ & 0.000 & 0.000 \\
\hline Ulnar nerve & $62.8 \pm 4.4$ & $57.4 \pm 3.2$ & $52.8 \pm 5.7^{\mathrm{bc}}$ & 0.000 & 0.000 \\
\hline Common peroneal nerve & $46.3 \pm 3.3$ & $45.3 \pm 2.8$ & $39.6 \pm 6.7^{b c}$ & 0.000 & 0.000 \\
\hline Tibial nerve & $47.3 \pm 3.1$ & $45.1 \pm 2.4$ & $39.5 \pm 6.0 \mathrm{bc}$ & 0.000 & 0.000 \\
\hline \multicolumn{6}{|l|}{ SCV } \\
\hline Median nerve & $51.8 \pm 6.0$ & $50.4 \pm 10.7$ & $46.5 \pm 6.3^{b c}$ & 0.146 & 0.000 \\
\hline Ulnar nerve & $57.2 \pm 3.1$ & $55.5 \pm 5.2$ & $52.4 \pm 4.4 \mathrm{bc}$ & 0.000 & 0.000 \\
\hline Sural nerve & $49.8 \pm 3.7$ & $47.8 \pm 3.1$ & $42.2 \pm 5.4$ bc & 0.000 & 0.000 \\
\hline \multicolumn{6}{|l|}{ Cognitive assessment } \\
\hline $\operatorname{MoCA}<26(n, \%)^{\#}$ & I, 2.8 & $11,30.6^{a}$ & $8,28.6^{b}$ & 0.005 & 1.000 \\
\hline MMSE & $29.2 \pm 0.8$ & $28.9 \pm 1.2$ & $28.4 \pm 1.5^{\mathrm{b}}$ & 0.032 & 0.109 \\
\hline $\mathrm{MoCA}$ & $28.0 \pm 1.6$ & $26.5 \pm 2.0^{\mathrm{a}}$ & $26.2 \pm 2.1^{\mathrm{b}}$ & 0.000 & 0.505 \\
\hline Memory & $206.9 \pm 22.6$ & $155.2 \pm 15.2^{\mathrm{a}}$ & $142 . \pm 20.0^{\mathrm{bc}}$ & 0.000 & 0.042 \\
\hline AVLT-immediate recall & $59.8 \pm 7.7$ & $39.4 \pm 6.1$ & $35.4 \pm 7.3$ & 0.159 & 0.257 \\
\hline AVLT-delayed recall (30 $\mathrm{min})$ & $17.1 \pm 3.1$ & $17.2 \pm 4.3$ & $15.2 \pm 4.1^{\mathrm{b}}$ & 0.081 & 0.043 \\
\hline AVLT-recognition & $57.4 \pm 1.9$ & $57.4 \pm 2.4$ & $56.8 \pm 2.8$ & 0.429 & 0.278 \\
\hline WASI-immediate recall & $19.3 \pm 5.8$ & $17.8 \pm 4.5$ & $15.8 \pm 5.0^{\mathrm{b}}$ & 0.029 & 0.126 \\
\hline WASI -delayed recall (30 min) & $15.5 \pm 6.5$ & $14.6 \pm 4.6$ & $13.1 \pm 5.0^{\mathrm{b}}$ & 0.217 & 0.284 \\
\hline WASI -recognition & $9.6 \pm 1.6$ & $8.8 \pm 1.9^{\mathrm{a}}$ & $8.8 \pm 1.8$ & 0.068 & 0.911 \\
\hline
\end{tabular}

(Continued) 
Table I (Continued).

\begin{tabular}{|c|c|c|c|c|c|}
\hline & HCs & NDPN & DPN & \multirow{2}{*}{$\begin{array}{c}P \text { value of } \\
\text { All }\end{array}$} & \multirow{2}{*}{$\begin{array}{l}P \text { value o } \\
\text { Diabetes }\end{array}$} \\
\hline & $n=36$ & $n=36$ & $\mathbf{n}=\mathbf{2 8}$ & & \\
\hline Working memory & $14.2 \pm 1.9$ & $13.1 \pm 1.8^{\mathrm{a}}$ & $13.1 \pm 2.4^{\mathrm{b}}$ & 0.035 & 0.967 \\
\hline DST-forward & $8.5 \pm 0.6$ & $8.3 \pm 0.9$ & $8.1 \pm 0.9^{b}$ & 0.063 & 0.562 \\
\hline DST-backward & $5.7 \pm 1.8$ & $4.8 \pm 1.2^{\mathrm{a}}$ & $5.0 \pm 1.8$ & 0.072 & 0.731 \\
\hline $\begin{array}{l}\text { Visual attention and task switching (processing } \\
\text { speed) }\end{array}$ & $20.7 \pm 4.3$ & $102.3 \pm 37.9^{\mathrm{a}}$ & $134.2 \pm 69.2^{\mathrm{bc}}$ & 0.000 & 0.004 \\
\hline TMT-A & $11.6 \pm 5.3$ & $13.9 \pm 6.4$ & $18 . \pm 10.1^{\mathrm{bc}}$ & 0.003 & 0.030 \\
\hline TMT-B & $87.7 \pm 35.9$ & $88.4 \pm 33.2$ & $116.2 \pm 61.6^{b c}$ & 0.019 & 0.014 \\
\hline \multicolumn{6}{|l|}{ Word fluency and retrieval performance } \\
\hline ANT & $20.8 \pm 4.5$ & $18.5 \pm 4.8^{\mathrm{a}}$ & $19.2 \pm 3.7$ & 0.090 & 0.537 \\
\hline BNT & $57.3 \pm 3.5$ & $56.6 \pm 2.6$ & $55.9 \pm 4.1$ & 0.264 & 0.395 \\
\hline Executive functions (time) & $66.7 \pm 20.1$ & $79.5 \pm 20.4^{\mathrm{a}}$ & $86.6 \pm 22.8^{b}$ & 0.041 & 0.056 \\
\hline SCWT-color & $17.4 \pm 5.1$ & $19.2 \pm 7.8$ & $22.0 \pm 6.2^{\mathrm{b}}$ & 0.023 & 0.085 \\
\hline SCWT-word & $20.2 \pm 7.8$ & $18.9 \pm 7.4$ & $24.1 \pm 10.0^{\mathrm{bc}}$ & 0.013 & 0.005 \\
\hline SCWT-color word & $29.0 \pm 9.0$ & $38.4 \pm 13.2^{\mathrm{a}}$ & $43.4 \pm 13.6$ & 0.016 & 0.187 \\
\hline \multicolumn{6}{|l|}{ Olfactory test } \\
\hline Total score & $50.2 \pm 5.4$ & $47.2 \pm 8.7$ & $43.5 \pm 6.0^{\mathrm{bc}}$ & 0.001 & 0.036 \\
\hline Olfactory threshold & $10.6 \pm 2.5$ & $8.6 \pm 3.1^{\mathrm{a}}$ & $8.4 \pm 3.0^{b}$ & 0.004 & 0.859 \\
\hline Odor identification test & $24.1 \pm 2.3$ & $23.6 \pm 4.3$ & $21.4 \pm 3.3^{b c}$ & 0.008 & 0.013 \\
\hline Task A (10 odors) & $8.6 \pm 1.1$ & $8.1 \pm 1.5$ & $7.6 \pm 1.7^{b}$ & 0.040 & 0.177 \\
\hline Task B (20 odors) & $15.5 \pm 1.8$ & $15.5 \pm 3.1$ & $13.8 \pm 2.1^{\mathrm{bc}}$ & 0.011 & 0.007 \\
\hline Odor memory test & $15.6 \pm 2.2$ & $15.0 \pm 3.1$ & $13.6 \pm 2.6^{\mathrm{bc}}$ & 0.015 & 0.041 \\
\hline Old 10 odors & $8.0 \pm 1.6$ & $7.7 \pm 1.6$ & $6.8 \pm 1.8^{\mathrm{bc}}$ & 0.011 & 0.029 \\
\hline New 10 odors & $7.6 \pm 1.6$ & $7.3 \pm 2.1$ & $6.9 \pm 2.0$ & 0.348 & 0.349 \\
\hline
\end{tabular}

Notes: Data are mean \pm standard deviation. Independent samples $t$-test between two groups. ${ }^{\#}$ Pearson chi-square analysis for dichotomous variables. ${ }^{a}$ Indicates $P<0.05$

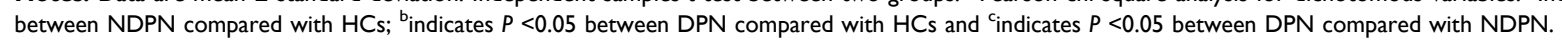

Abbreviations: HbAlc, glycated hemoglobin; BMI, body mass index; WHR, waist-hip ratio; MMSE, Mini-Mental State Examination; MoCA, Montreal Cognitive Assessment. ANT, Animal Naming Test; AVLT, Auditory Verbal Learning Test; BNT, Boston Naming Test; DST, Digit Span Task; SCWT, Stroop Color and Word Test; TMT, Trail Making Test; WASI, Wechsler Abbreviated Scale of Intelligence.

\section{Odor-Induced-Task fMRI and Seed-Based}

\section{Functional Connectivity}

The olfactory-related regions of the brain were bilaterally activated after odor stimulation (Figure 1A and B). The independent-sample $t$-test showed reduced activation in the left frontal lobe in patients with DPN compared with that in the NDPN group (cluster size threshold: 51 voxels, with GRF correction, voxel level: $\mathrm{P}<0.001$, cluster level: $\mathrm{P}<0.05$,) (Figure 1C-E). Positive associations were detected between activation of the left frontal lobe and sensory activation of the ulnar nerve and sural nerve (Figure 2).

As shown in Figure 3, the independent-sample $t$-test revealed significantly decreased seed-based FC in the right insula in the DPN group compared with the NDPN group (cluster size threshold: 129 voxels, with GRF correction; voxel level: $\mathrm{P}<0.001$, cluster level: $\mathrm{P}<0.05$ ).

\section{Structural Equation Analysis for Modelling the Interactions Among Neuropathy Parameters, Cognition, and Olfaction}

The path model (Figure 4) showed satisfactory model fit statistics, with $\mathrm{CMIN} / \mathrm{DF}=1.510$, CFI $=0.971$, NFI $=$ 0.926 , TLI $=0.912$, and RMSEA $=0.071$. The results show that the SCV of the median nerve is directly associated with the score for olfactory identification $(\beta=0.349, p=$ $0.006,95 \% C I=0.108-0.563)$ and executive function $(\beta=$ $-0.266, p=0.009,95 \% C I=-0.449--0.067)$. The score for olfactory identification were negatively associated with the executive function consumption $(\beta=-0.367, p=$ $0.002,95 \% C I=-0.585--0.140$ ). Given that there was a direct association between the SCV and executive function, the Sobel test of indirect effect confirmed that olfactory identification partially mediated the association between the SCV and executive function $(\beta=-0.128$, 


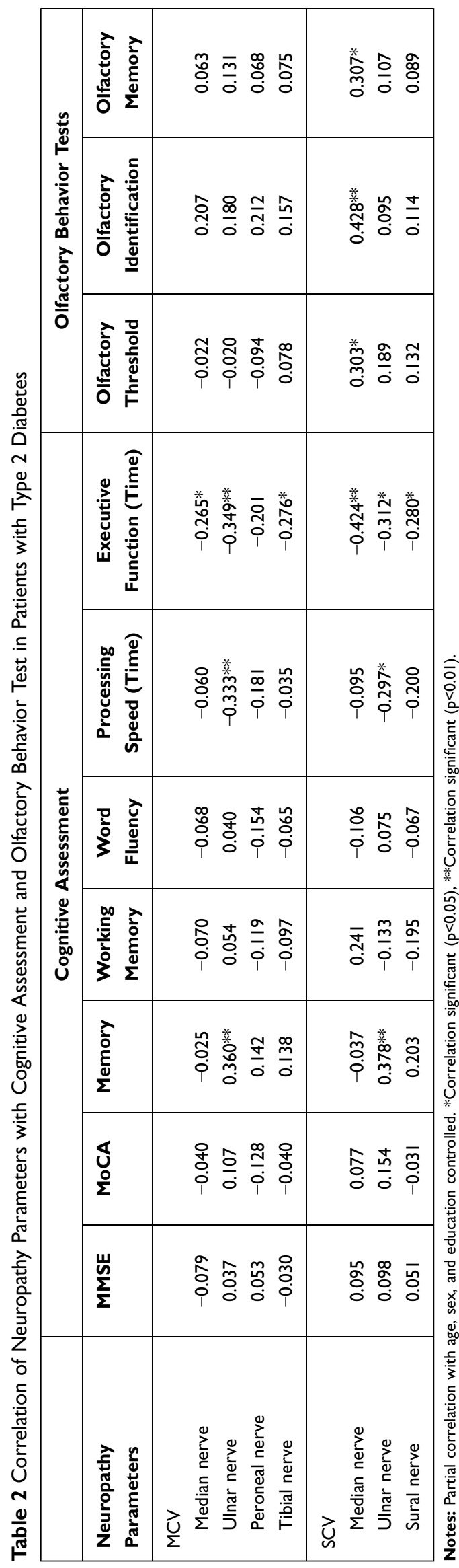

$p=0.003,95 \% C I=-0.278-0.039)$. Effect size calculations using MacKinnon's formula (ie, the indirect effect: $\beta$ $=-0.128$ divided by the total effect: $\beta=-0.395$ ) showed that the partial mediated percentage was $32 \%$ representing a small effect size according to Cohen's guidelines.

\section{Discussion}

We demonstrated that patients with DPN, when compared to those without DPN, suffered a decline in cognitive domains in memory and processing speed that correlated with the NCV. Moreover, the patients with DPN exhibited impaired olfactory behavior and decreases in activation in the left frontal lobe and in seed-based functional connectivity in the right insula. Furthermore, the results of the electrophysiological examination, combined with the observed olfactory behavior, could predict cognitive decline in T2DM patients.

Patients with DPN suffered a decline in the cognitive domains of memory and processing speed. Recently, several studies have reported results for cognition in T2DM patients. ${ }^{1}$ Diabetes-associated cognitive decrements have been reported to develop approximately $50 \%$ faster than those observed in normal cognitive aging. ${ }^{19}$ However, few studies have focused on cognition in DPN. Moreira and colleagues showed that cognitive impairment does not seem to have a relationship with the presence and severity of DPN; however, only a neurologic deficit scale (NDS) and a neurological severity score (NSS) were used for the diagnosis of DPN, while cognitive function was assessed using MMSE, the Trail Making Test (part A), the Trail Making Test (part B), and the Verbal Fluency TestAnimals. ${ }^{3}$ By contrast, we measured the SCV and MCV of the upper and lower limbs, as well as the general cognitive function and detailed cognitive domains. We demonstrated, for the first time, that patients with DPN had a worse cognitive memory function and processing speed than was observed in patients without DPN, and that the NCVs were related to several cognitive domains.

Olfactory dysfunction is viewed as a potential and early indicator for the diagnosis of neurodegenerative disorders, ${ }^{20}$ as it predicts incident mild cognitive impairment. $^{21}$ In addition, reduced olfactory-threshold scores have been found in T2DM patients with apparently normal cognition, ${ }^{15}$ and Gouveri and colleagues found that olfactory dysfunction was associated with retinopathy. ${ }^{22}$ We have provided new evidence that patients with DPN had worse odor identification and poorer memory than patients who did not have DPN, and the DPN effects had 


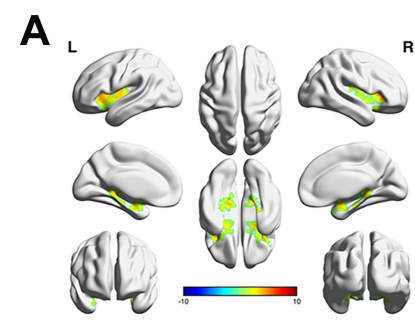

DPN

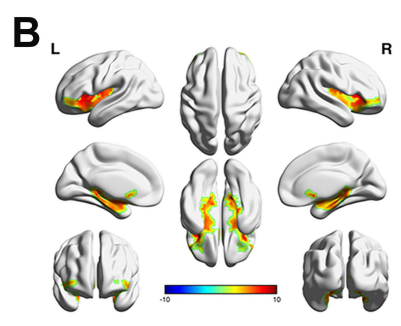

NDPN

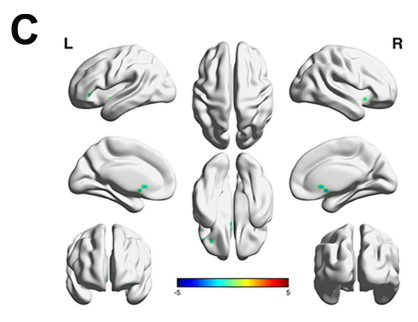

DPN - NDPN
D

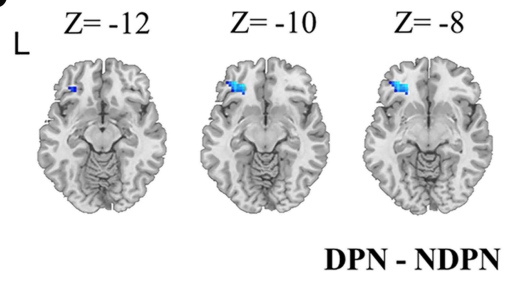

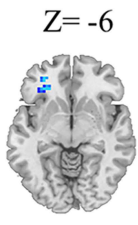

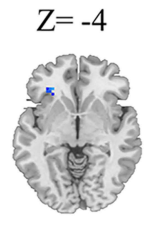

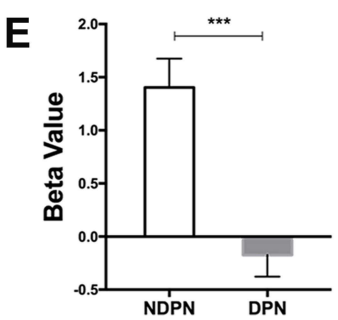

Figure I Comparison of odor-induced brain activation in DPN and NDPN. Olfactory-related regions of the brain were activated after odor stimulation in DPN (A) and NDPN (B). Significantly decreased brain activation was demonstrated in DPN compared to NDPN (with GRF correction, voxel level: P < 0.001 , cluster level: P < 0.05 , cluster size threshold: 5 I voxels) (C). $\beta$ value is decreased in the left frontal lobe in DPN (D and E). Independent sample $t$ test. $* * * P<0.00 I$. L, left; $\mathrm{R}$, right.

A

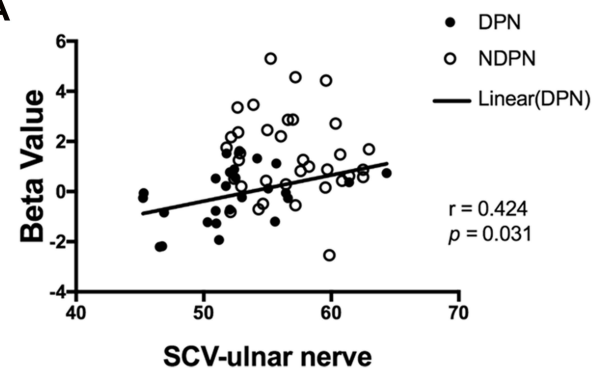

B

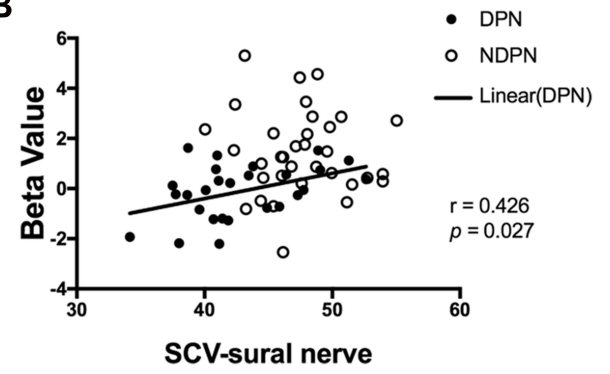

Figure 2 Associations between odor-induced brain activation and nerve conduction. Positive associations between the activation of the left frontal lobe and sensory nerve conduction of ulnar nerve (A) and sural nerve (B) in DPN group.

Abbreviation: SCV, sensory conduction velocity.

a positive correlation with NCVs. Our use of computerized and standardized olfactory tests instead of Sniffin' Sticks made our data more robust.

Emerging evidence from MRI and fMRI studies has shown an impact of DPN on the structure and function of the brain. ${ }^{23}$ People suffering from DPN who show lower gray-matter volume (globally and regionally) also walk more slowly and have greater variations in stride duration and/or longer support. ${ }^{24}$ Recent fMRI research has revealed an association between the poor functional performance in patients with DPN and greater activation in motor preparation regions. ${ }^{25}$ Similarly, MRI findings have revealed an involvement of the central nervous system, including dysfunction of somatosensory afferent pathways, in DPN. ${ }^{26,27}$ In addition, approximately $50 \%$ of patients with DPN can experience painful neuropathic symptoms, which are the most distressing symptoms of DPN. ${ }^{28,29}$ A number of central mechanisms, including central sensitization, changes in the balance of facilitation/inhibition within descending pathways, and increased thalamic vascularity, have recently been proposed based on experiments on neuropathic pain. ${ }^{23,30}$ The dorsal root ganglion has been considered as a treatment target for neuropathic pain because of its structure at the communication point in the transition from the peripheral to the central nervous system. ${ }^{31}$ However, few studies have focused on olfactory-related regions and functional connectivity. We have revealed a reduced activation in the left frontal lobe and a reduction in seed-based functional connectivity in the right insula in patients with DPN compared with those without DPN. These findings indicate a connection between the activation of olfactory neural circuits and DPN. 


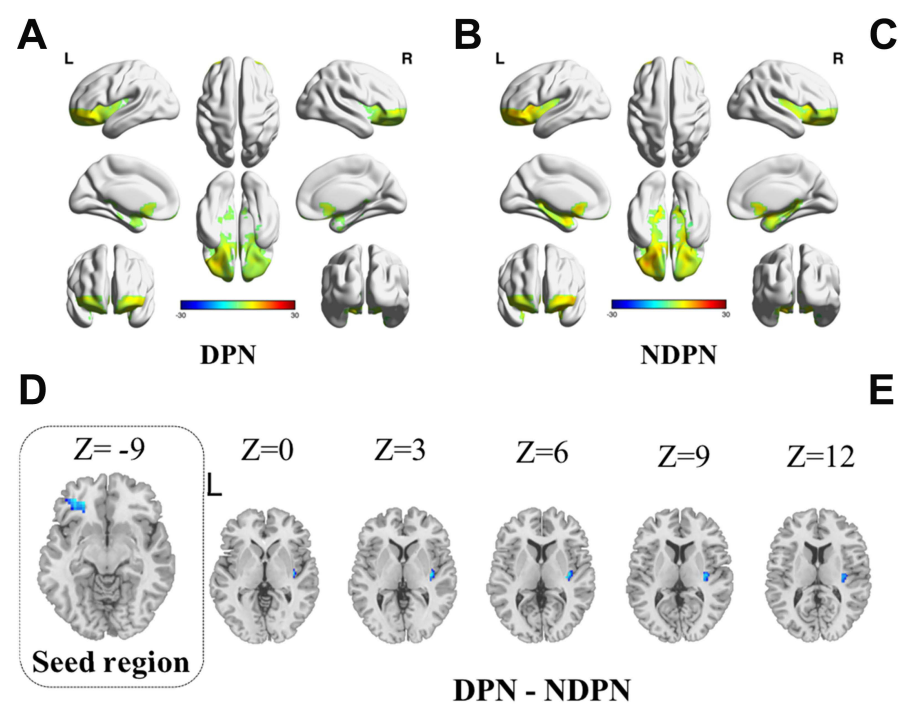

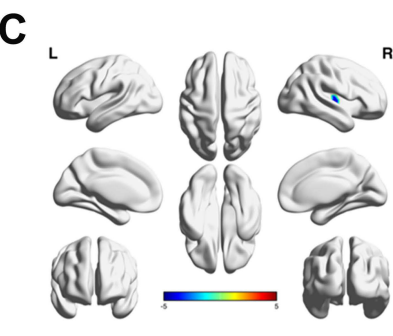

DPN - NDPN

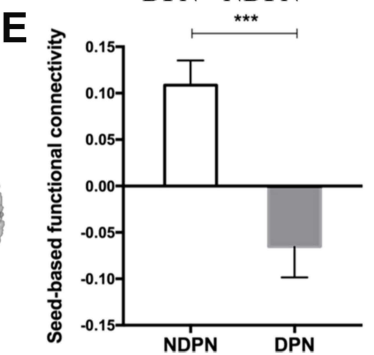

Figure 3 Comparison of seed-based functional connectivity in DPN and NDPN. The seed-based functional connectivity was shown in DPN (A) and NDPN (B). Significantly decreased brain functional connectivity was demonstrated in DPN compared to NDPN (with GRF correction, voxel level: $P<0.001$, cluster level: $\mathrm{P}<0.05$, cluster size threshold: 129 voxels) (C). Seed-based functional connectivity with right insula is decreased in DPN (D and E). Independent sample $t$ test. $* * * P<0.00$ I. L, left; R, right.

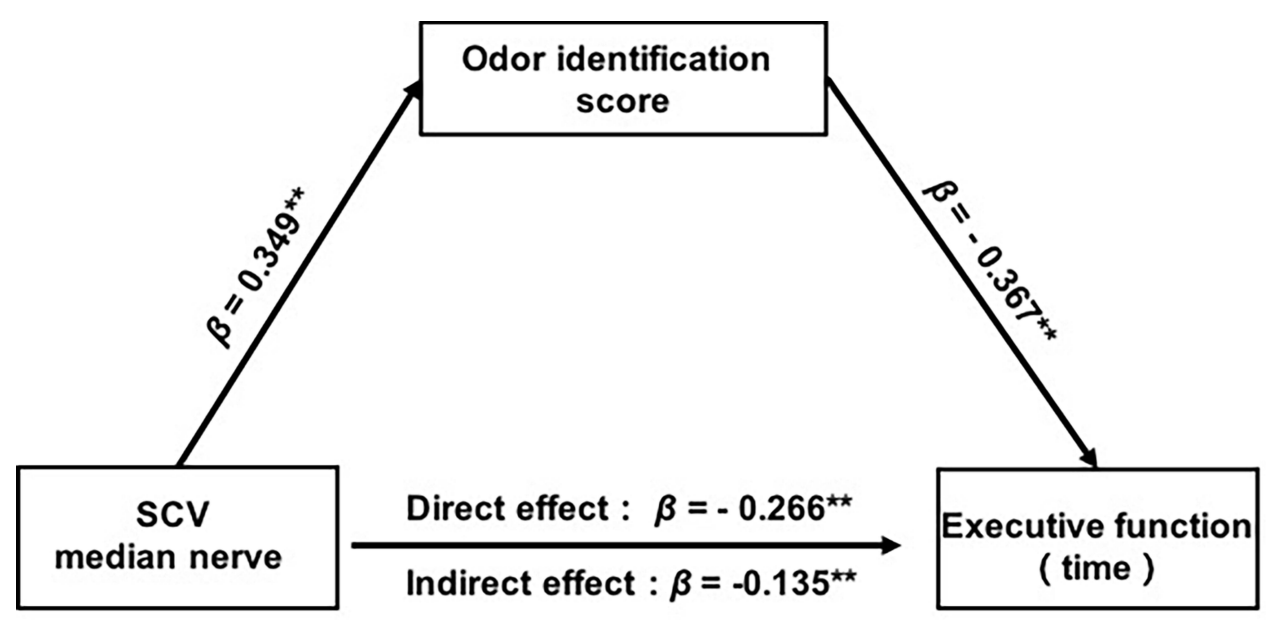

Figure 4 Structual model among cognition, olfactory behavior and neuropathy parameters. Adjusted mediation model predicting executive function among diabetes with DPN. Analyses are adjusted for age, sex, and education. ${ }^{* * p}<0.0 \mathrm{I}, \rightarrow$ pathway.

We observed that conduction in the ulnar nerve was correlated significantly with cognition and olfactory behavior in T2DM patients. Olfactory identification acted as a mediating factor in the effect of $\mathrm{NCV}$ on executive function, suggesting an involvement of olfactory function in the cognitive impairment in DPN. Peripheral nerves, including the trigeminal nerve, glossopharyngeal nerve, and vagus nerve, in addition to the olfactory nerve, are involved in the formation of the olfactory system. ${ }^{32}$ The olfactory neural circuits in the hippocampus are also connected with cognition, including memory processes. ${ }^{33}$ Our findings suggest that olfactory dysfunction may have a role in the pathogenesis of peripheral neuropathy and cognitive impairment, and that declines in olfactory capabilities could be indicators of cognitive decline in patients with DPN.

Our study had two main limitations. One was that the sample size was relatively small. Nevertheless, our patients were well matched for age, sex, and educational level, and we believe our study could provide insights into central changes in DPN patients. The second limitation was its cross-sectional design, which precludes any comments on the causal relationship between DPN and olfactory dysfunction in patients with DPN. Follow-up studies are warranted to ascertain the role of olfactory dysfunction in DPN and to determine if olfactory dysfunction could be an early sign of DPN and cognitive impairment. 


\section{Conclusion}

This was the first study to demonstrate that patients with DPN had worse cognitive function in the domains of memory and processing speed compared to patients without DPN. Examinations of nerve conduction, combined with olfactory tests, might therefore aid in the early detection of cognitive impairment in patients with T2DM. Greater attention should be focused on screening for cognitive impairment in patients with DPN.

\section{Abbreviations}

DPN, diabetic peripheral neuropathy; T2DM: type 2 diabetes mellitus; magnetic resonance imaging (MRI); fMRI, functional magnetic resonance imaging; $\mathrm{FC}$, functional connectivity; MCI, mild cognitive impairment; NCV, nerve conduction velocity; HbAlc, glycosylated hemoglobin; TC, total cholesterol; TG, triglyceride; HDL-C, high-density lipoprotein-cholesterol; LDL-C, low-density lipoprotein cholesterol; ROI, regions of interest; GRF, Gaussian random field; MCV, motor conduction velocity; $\mathrm{SCV}$, sensory conduction velocity; NDS, neurologic deficit scale; NSS, neurological severity score; BMI, body mass index; WHR, waist-hip ratio; MMSE, Mini-Mental State Examination; MoCA, Montreal Cognitive Assessment. ANT, Animal Naming Test; AVLT, Auditory Verbal Learning Test; BNT, Boston Naming Test; DST, Digit Span Task; SCWT, Stroop Color and Word Test; TMT, Trail Making Test; WASI, Wechsler Abbreviated Scale of Intelligence; SEM, Structural Equation Modeling; RMSEA, Root Mean Square Error of Approximation; NFI, Normal Fit Index; CFI, Comparative Fix Index; TLI, Tucker-Lewis Index.

\section{Human Rights}

All procedures performed in studies involving human participants were in accordance with the ethical standards of the institutional research committee and with the 1964 Helsinki Declaration and its later amendments or comparable ethical standards.

\section{Data Sharing Statements}

The data that support the findings of this study are available from the corresponding author upon reasonable request.

\section{Ethical Approval}

Our survey was approved by the Ethics Review Committee of Drum Tower Hospital. The registered clinical trial number on ClinicalTrails.gov is NCT 02738671.

\section{Informed Consent}

All participants provided written informed consent prior to their inclusion in the study.

\section{Acknowledgments}

The authors would like to thank all volunteers for their participation in this study and thank medical personnel from Department of Endocrinology and Department of Radiology, Drum Tower Hospital affiliated to Nanjing University Medical School, for their valuable assistance. Wenyu Ni and Zhou Zhang shared first authorship.

\section{Funding}

This work was supported by the National Natural Science Foundation of China Grant Awards (82000775, 82030026, 81970689, 81970704, 81770819, 81703294, 81800752, 81900787, and 81800719), the National Key Research and Development Program of China (2016YFC1304804 and 2017YFC1309605), the Jiangsu Provincial Key Medical Discipline (ZDXKB2016012), the Key Project of Nanjing Clinical Medical Science, the Key Research and Development Program of Jiangsu Province of China (BE2015604 and BE2016606), the Jiangsu Provincial Medical Talent (ZDRCA2016062), the Six Talent Peaks Project of Jiangsu Province of China (YY-086), the Scientific Research Project of the Fifth Phase of "333 Project" of Jiangsu Province of China, the Fundamental Research Funds for the Central Universities (021414380444).

\section{Disclosure}

The authors report no conflicts of interest in this work.

\section{References}

1. Livingston G, Huntley J, Sommerlad A, et al. Dementia prevention, intervention, and care: 2020 report of the lancet commission. Lancet. 2020;396(10248):413-446. doi:10.1016/S0140-6736(20)30367-6

2. Jia L, Du Y, Chu L, et al. Prevalence, risk factors, and management of dementia and mild cognitive impairment in adults aged 60 years or older in China: a cross-sectional study. Lancet Public Health. 2020;5 (12):e661-e671. doi:10.1016/s2468-2667(20)30185-7

3. Biessels GJ, Despa F. Cognitive decline and dementia in diabetes mellitus: mechanisms and clinical implications. Nat Rev Endocrinol. 2018;14(10):591-604. doi:10.1038/s41574-018-0048-7

4. Livingston G, Sommerlad A, Orgeta V, et al. Dementia prevention, intervention, and care. Lancet. 2017;390(10113):2673-2734. doi:10.1016/S0140-6736(17)31363-6

5. Feldman E, Callaghan B, Pop-Busui R, et al. Diabetic neuropathy. Nat Rev Dis Primers. 2019;5(1):41. doi:10.1038/s41572-019-0092-1

6. Tesfaye S, Boulton AJ, Dyck PJ, et al. Diabetic neuropathies: update on definitions, diagnostic criteria, estimation of severity, and treatments. Diabetes Care. 2010;33(10):2285-2293. doi:10.2337/ dc10-1303 
7. Boulton AJM, Vileikyte L, Ragnarson-Tennvall G, Apelqvist J. The global burden of diabetic foot disease. Lancet. 2005;366 (9498):1719-1724. doi:10.1016/S0140-6736(05)67698-2

8. Selvarajah D, Kar D, Khunti K, et al. Diabetic peripheral neuropathy: advances in diagnosis and strategies for screening and early intervention. The lancet. Diabetes \& Endocrinology. 2019;7 (12):938-948. doi:10.1016/s2213-8587(19)30081-6

9. Moreira RO, Soldera AL, Cury B, Meireles C, Kupfer R. Is cognitive impairment associated with the presence and severity of peripheral neuropathy in patients with type 2 diabetes mellitus? Diabetol Metab Syndr. 2015;7.

10. Roberts RO, Knopman DS, Geda YE, et al. Association of diabetes with amnestic and nonamnestic mild cognitive impairment. Alzheimers Dement. 2014;10(1):18-26. doi:10.1016/j. jalz.2013.01.001

11. Roberts RO, Christianson TJH, Kremers WK, et al. Association between olfactory dysfunction and amnestic mild cognitive impairment and alzheimer disease dementia. JAMA Neurol. 2016;73 (1):93-101. doi:10.1001/jamaneurol.2015.2952

12. Dintica CS, Marseglia A, Rizzuto D, et al. Impaired olfaction is associated with cognitive decline and neurodegeneration in the brain. Neurology. 2019;92(7):e700-e709. doi:10.1212/ wnl.0000000000006919

13. Mahlknecht P, Iranzo A, Hogl B, et al. Olfactory dysfunction predicts early transition to a Lewy body disease in idiopathic RBD. Neurology. 2015;84(7):654-658. doi:10.1212/ Wnl.0000000000001265

14. Devanand DP, Tabert MH, Cuasay K, et al. Olfactory identification deficits and MCI in a multi-ethnic elderly community sample. Neurobiol Aging. 2010;31(9):1593-1600. doi:10.1016/j. neurobiolaging.2008.09.008

15. Zhang Z, Zhang B, Wang X, et al. Altered Odor-Induced Brain Activity as an Early Manifestation of Cognitive Decline in Patients With Type 2 Diabetes. Diabetes. 2018;67(5):994-1006. doi:10.2337/ db17-1274

16. Zhang Z, Zhang B, Wang X, et al. Olfactory dysfunction mediates adiposity in cognitive impairment of type 2 diabetes: insights from clinical and functional neuroimaging studies. Diabetes Care. 2019;42 (7):1274-1283. doi:10.2337/dc18-2584

17. Bronzino JD, ed. Electromyography Diagnosis and Clinical Application. People's Medical Publishing House; 2013.

18. Nasreddine ZS, Phillips NA, Bedirian V, et al. The montreal cognitive assessment, MoCA: a brief screening tool for mild cognitive impairment. J Am Geriatr Soc. 2005;53(4):695-699. doi:10.1111/ j.1532-5415.2005.53221.x

19. Preacher KJ, Hayes AF. Asymptotic and resampling strategies for assessing and comparing indirect effects in multiple mediator models. Behav Res Methods. 2008;40(3):879-891. doi:10.3758/brm.40.3.879

20. Moreira RO, Soldera AL, Cury B, Meireles C, Kupfer R. Is cognitive impairment associated with the presence and severity of peripheral neuropathy in patients with type 2 diabetes mellitus? Diabetol Metab Syndr. 2015;7(1):51. doi:10.1186/s13098-015-0045-0
21. Doty RL. Olfactory dysfunction in neurodegenerative diseases: is there a common pathological substrate? Lancet Neurol. 2017;16 (6):478-488. doi:10.1016/s1474-4422(17)30123-0

22. Conti MZ, Vicini-Chilovi B, Riva M, et al. Odor identification deficit predicts clinical conversion from mild cognitive impairment to dementia due to Alzheimer's disease. Arch Clin Neuropsychol. 2013;28(5):391-399. doi:10.1093/arclin/act032

23. Gouveri E, Katotomichelakis M, Gouveris H, Danielides V, Maltezos E, Papanas N. Olfactory dysfunction in type 2 diabetes mellitus: an additional manifestation of microvascular disease? Angiology. 2014;65(10):869-876. doi:10.1177/0003319714520956

24. Tesfaye S, Selvarajah D, Gandhi R, et al. Diabetic peripheral neuropathy may not be as its name suggests: evidence from magnetic resonance imaging. Pain. 2016;157(Suppl 1):S72-80. doi:10.1097/j. pain.0000000000000465

25. Manor B, Newton E, Abduljalil A, Novak V. The relationship between brain volume and walking outcomes in older adults with and without diabetic peripheral neuropathy. Diabetes Care. 2012;35 (9):1907-1912. doi:10.2337/dc11-2463

26. Venkataraman K, Pun V, Mohamed AZ, et al. Altered motor and motor perceptual cognitive imagery task-related activation in diabetic peripheral neuropathy: insights from functional MRI. Diabetes Care. 2019;42(10):2004-2007. doi:10.2337/dc19-0746

27. Selvarajah D, Wilkinson ID, Gandhi R, Griffiths PD, Tesfaye S. Microvascular perfusion abnormalities of the Thalamus in painful but not painless diabetic polyneuropathy: a clue to the pathogenesis of pain in type 1 diabetes. Diabetes Care. 2011;34(3):718-720. doi: $10.2337 / \mathrm{dc} 10-1550$

28. Selvarajah D, Wilkinson ID, Fang F, et al. Structural and functional abnormalities of the primary somatosensory cortex in diabetic peripheral neuropathy: a multimodal MRI Study. Diabetes. 2019;68 (4):796-806. doi:10.2337/db18-0509

29. Griebeler M, Morey-Vargas O, Brito J, et al. Pharmacologic interventions for painful diabetic neuropathy: an umbrella systematic review and comparative effectiveness network meta-analysis. Ann Intern Med. 2014;161(9):639-649. doi:10.7326/m14-0511

30. Saive AL, Royet JP, Plailly J. A review on the neural bases of episodic odor memory: from laboratory-based to autobiographical approaches. Front Behav Neurosci. 2014;8(1662-5153 (Print)). doi:10.3389/fnbeh.2014.00240

31. Tesfaye S, Kempler P. Painful diabetic neuropathy. Diabetologia. 2005;48(5):805-807. doi:10.1007/s00125-005-1721-7

32. Liem L, van Dongen E, Huygen F, Staats P, Kramer JJ. The dorsal root ganglion as a therapeutic target for chronic pain. Reg Anesth Pain Med. 2016;41(4):511-519. doi:10.1097/aap.0000000000000408

33. Landis BN, Scheibe M, Weber C, et al. Chemosensory interaction: acquired olfactory impairment is associated with decreased taste function. J Neurol. 2010;257(8):1303-1308. doi:10.1007/s00415010-5513-8

\section{Publish your work in this journal}

Diabetes, Metabolic Syndrome and Obesity: Targets and Therapy is an international, peer-reviewed open-access journal committed to the rapid publication of the latest laboratory and clinical findings in the fields of diabetes, metabolic syndrome and obesity research. Original research, review, case reports, hypothesis formation, expert opinion and commentaries are all considered for publication. The manuscript management system is completely online and includes a very quick and fair peer-review system, which is all easy to use. Visit http://www.dovepress.com/testimonials.php to read real quotes from published authors. 